\title{
Palliative Radiation Therapy for Macroscopic Hematuria Caused by Urothelial Cancer
}

\author{
Haiqin Zhang, MD, ${ }^{1-3}$ Hidehiro Hojo, MD, PhD, ${ }^{3, *}$ Vijay Parshuram Raturi, MD, ${ }^{3}$ Naoki Nakamura, MD, PhD, ${ }^{3}$ \\ Masaki Nakamura, MD, Masayuki Okumura, MD, ${ }^{3}$ Yasuhiro Hirano, MD, ${ }^{3}$ Atsushi Motegi, MD, PhD, \\ Shun-Ichiro Kageyama, MD, $\mathrm{PhD}^{3}$ Sadamoto Zenda, MD, $\mathrm{PhD}^{3}$ and Tetsuo Akimoto, MD, $\mathrm{PhD}^{3}$
}

\begin{abstract}
Background: To assess the efficacy and toxicity profiles of palliative radiation therapy (RT) for macroscopic hematuria (MH) caused by urothelial cancer.

Methods: A total of 25 urothelial cancer patients with MH who underwent palliative RT between 2008 and 2018 were analyzed in this retrospective study. The hematuria-free survival (HFS) time was defined as the period from complete resolution of $\mathrm{MH}$ to the recurrence of $\mathrm{MH}$, death, or the last follow-up examination. Adverse events were classified according to the Common Terminology Criteria for Adverse Events version 4.0.

Results: By the end of the median follow-up duration of 90 days (11-886 days), complete resolution of MH had been achieved in 22 patients (88\%), and the median interval between the start of RT and resolution of MH was 9 days (2-179 days). Of the 22 patients in whom the symptom resolved, 9 (41\%) developed recurrent $\mathrm{MH}$, and the median time to relapse of MH was 129 days (30-692 days). The median RT dose was 30 Gy (20-40 Gy). Nine (36\%) patients received a blood transfusion before the RT. The three-month HFS rate was $52.1 \%$. There was a significant difference in the three-month HFS rate between patients with and without a history of pretreatment blood transfusion (HFS rate: $34.6 \%$ vs. $61.5 \%, p=0.03$ ). Grade 2 urinary tract pain and grade 3 diarrhea were seen in one patient each.
\end{abstract}

Conclusion: Palliative RT appeared to be effective with limited toxicities for urothelial cancer patients with MH.

Keywords: hematuria; palliative radiation therapy; urothelial cancer

\section{Introduction}

Urothelial carcinoma (UC) is one of the most commonly occurring tumors in the world, especially in developed countries. Over half of the patients with UC show recurrence or tumor progression even after definitive treatments. ${ }^{1}$ In patients with UC, hematuria, especially macroscopic hematuria $(\mathrm{MH})$, is the most common presenting symptom; it is often intractable, adversely affecting the quality of life of the patients. Uncontrollable $\mathrm{MH}$ is sometimes fatal. Several treatment approaches have been suggested, limited by poor treatment compliance or absence of proven effectiveness, including intravesical formalin treatment, alum irrigation, intravesical instillation of prostaglandin, hydrostatic bladder distention, urinary diversion, intra-arterial chemoperfusion with mitoxantrone, and embolization. ${ }^{2}$

Palliative radiotherapy (RT) is sometimes used as a treatment option for hematuria, with the reported hematuria improvement rate varying from $54 \%$ to $92 \%{ }^{3-7} \mathrm{~A}$ few reports of retrospective analyses of palliative RT for hematuria in patients with UCs have been published,

\footnotetext{
${ }^{1}$ Department of Oncology, Jinan Central Hospital, Cheeloo College of Medicine, Shandong University, Jinan, Shandong, P.R. China.

${ }^{2}$ Department of Oncology, Jinan Central Hospital Affiliated to Shandong First Medical University, Jinan, Shandong, P.R. China.

${ }^{3}$ Division of Radiation Oncology and Particle Therapy, National Cancer Center Hospital East, Kashiwa, Chiba, Japan.

*Address correspondence to: Hidehiro Hojo, MD, PhD, Division of Radiation Oncology and Particle Therapy, National Cancer Center Hospital East, 6-5-1, Kashiwanoha, Kashiwa, Chiba, 277-8577, Japan, E-mail: hhojo@east.ncc.go.jp
}

(c) Haiqin Zhang et al., 2020; Published by Mary Ann Liebert, Inc. This Open Access article is distributed under the terms of the Creative Commons License (http://creativecommons.org/licenses/by/4.0), which permits unrestricted use, distribution, and reproduction in any medium, provided the original work is properly cited. 
and most are based on analyses of small cohorts. In 2016, a survey conducted by the palliative RT working group of the Japanese Radiation Oncology Study Group suggested that hemostatic irradiation is rarely performed at facilities in Japan and further that the number of fractions used varied significantly among facilities. ${ }^{8}$

Hence, the purpose of this study was to conduct a retrospective analysis of the efficacy and toxicity of palliative RT for $\mathrm{MH}$ in patients with urothelial cancer at our institution.

\section{Materials and Methods}

Ethics statement

This retrospective study was conducted with the approval of the ethics committee of our institution (approval number: 2017-440). The study was conducted in accordance with the Declaration of Helsinki.

\section{Patients}

The study was conducted in 25 urothelial cancer patients with $\mathrm{MH}$ who received palliative RT between 2008 and 2018 at the National Cancer Center Hospital East. MH is defined as blood in the urine that can be seen with the naked eye. The patients were retrospectively enlisted from our database, and patients in whom the $\mathrm{MH}$ was caused by direct bleeding from the bladder tumor, which was established based on the clinical and/or diagnostic imaging findings, were analyzed. The clinical tumor stage (Union for International Cancer Control, eighth edition) was determined based on the findings of contrast-enhanced computed tomography and/or magnetic resonance imaging.

During the simulation, all patients were instructed to have an empty bladder and rectum. The clinical target volume (CTV) was the whole bladder, and in some patients, it included a part of the ureter, if the patient had been diagnosed as having ureteric invasion. The planning target volume was determined by the isotropic expansion of the CTV with a margin of $0.5-2 \mathrm{~cm}$ based on movement of the bladder in each patient. The four-field technique was adopted. Complete resolution of $\mathrm{MH}$ after RT was deemed as representing treatment success, while reappearance of $\mathrm{MH}$ was defined as relapse. Adverse events were classified according to the Common Terminology Criteria for Adverse Events version 4.0.

\section{Statistical analyses}

EZR version 1.37 was used to perform all the statistical analyses. ${ }^{9}$ The hematuria-free survival (HFS) time was defined as the period from complete resolution of $\mathrm{MH}$ to the recurrence of $\mathrm{MH}$, death, or the last follow-up examination. Chi-square test or Fisher's exact test was used to determine the significance of intergroup differences in discontinuous variables. The Kaplan-Meier method was used to evaluate survival probability. The survival between different groups was compared using the logrank test. Univariate analysis and multivariate analysis were performed using the Cox regression model, with factors identified as significant by the former $(p<0.25)$ being entered into the latter model. The cutoff values of age, hemoglobin, and creatinine for analysis were set to the median values. The HFS in the multivariate analysis was performed using Akaike's information criterion (AIC) and Bayesian information criterion (BIC) methods. The Jonckheere-Terpstra test was performed to test for the trend in the period from the first day of treatment to the day of resolution of hematuria. $p<0.05$ was considered being indicative of statistical significance. The variables used for the statistical analyses were age, gender, Eastern Cooperative Oncology Group (ECOG) performance status (PS), hemoglobin levels just before the radiotherapy, serum creatinine, the Union for International Cancer Control (UICC) tumor class, history of pretreatment blood transfusion, and history of chemotherapy before the palliative RT.

\section{Results}

The characteristics of the 25 patients included in the analysis are shown in Table 1 . The study population included 15 males and 10 females, with a median age of 73 years (range: $57-91$ years); 16 patients $(64 \%)$ had an ECOG PS of 0 to 1 . The dominant histology was transitional cell carcinoma ( 22 patients; $88 \%$ ), and the primary site of the tumor was the bladder in 21 patients (84\%). Of the 25 patients, 24 were not suitable candidates for radical surgery because of advanced age ( 9 ; $36 \%)$, poor general condition $(3 ; 12 \%)$, widespread metastasis $(3 ; 12 \%)$, recurrence after transurethral resection of bladder tumor $(7 ; 28 \%)$, or patient's refusal to undergo definitive treatment $(3 ; 12 \%)$.

Fourteen patients had a history of chemotherapy before irradiation. No patient whose interval between the last day of chemotherapy and the start of RT was less than 25 days was found (median duration 27 days; 25237 days). After palliative RT, one patient underwent chemotherapy, one patient underwent reirradiation for recurrent hematuria, and one patient underwent chemotherapy followed by reirradiation. The remaining patients did 
Table 1. Patient Characteristics

\begin{tabular}{|c|c|}
\hline & No. of patients \\
\hline Median age (range) & 73 years $(57-91)$ \\
\hline \multicolumn{2}{|l|}{ Gender } \\
\hline Male & 15 \\
\hline Female & 10 \\
\hline \multicolumn{2}{|l|}{ PS (ECOG) } \\
\hline $0-1$ & 16 \\
\hline$\geq 2$ & 9 \\
\hline \multicolumn{2}{|l|}{ Histological type } \\
\hline TCC & 22 \\
\hline Adenocarcinoma & 1 \\
\hline Squamous & 1 \\
\hline NEC & 1 \\
\hline \multicolumn{2}{|l|}{ Primary site } \\
\hline Bladder & 21 \\
\hline Ureter & 3 \\
\hline Allantoic duct & 1 \\
\hline \multicolumn{2}{|l|}{ UICC T classifications } \\
\hline $\mathrm{T} 2$ & 10 \\
\hline T3 & 8 \\
\hline $\mathrm{T} 4$ & 7 \\
\hline \multicolumn{2}{|l|}{ Node classifications } \\
\hline Negative & 17 \\
\hline Positive & 8 \\
\hline \multicolumn{2}{|l|}{ Distant metastases } \\
\hline Yes & 3 \\
\hline No & 22 \\
\hline \multicolumn{2}{|l|}{ Hemoglobin (g/dL) } \\
\hline$<9.3$ & 11 \\
\hline$\geq 9.3$ & 13 \\
\hline Unknown & 1 \\
\hline \multicolumn{2}{|l|}{ Creatinine (mg/dL) } \\
\hline$<0.95$ & 11 \\
\hline$\geq 0.95$ & 13 \\
\hline Unknown & 1 \\
\hline \multicolumn{2}{|l|}{ Irradiation dose } \\
\hline $20 \mathrm{~Gy} / 5 \mathrm{fr}$ & 7 \\
\hline $30 \mathrm{~Gy} / 10 \mathrm{fr}$. & 17 \\
\hline $40 \mathrm{~Gy} / 20 \mathrm{fr}$. & 1 \\
\hline \multicolumn{2}{|c|}{ History of pretreatment blood transfusion } \\
\hline Yes & 9 \\
\hline No & 16 \\
\hline \multicolumn{2}{|c|}{ History of chemotherapy before irradiation } \\
\hline Yes & 14 \\
\hline No & 11 \\
\hline
\end{tabular}

ECOG, Eastern Cooperative Oncology Group; fr, fractions; NEC, neuroendocrine carcinoma; PS, performance status; TCC, transitional cell carcinoma; UICC, Union for International Cancer Control.

not undergo any treatment intervention after palliative RT . The median hemoglobin level before palliative RT was $9.3 \mathrm{~g} / \mathrm{dL}$ (range: $5.1-15.5 \mathrm{~g} / \mathrm{dL}$ ). The median hemoglobin level in the $9(36 \%)$ patients who had received blood transfusion before the RT was $8.3 \mathrm{~g} / \mathrm{dL}$ (range: $5.1-11 \mathrm{~g} / \mathrm{dL}$ ), while that in the remaining $16(64 \%)$ patients was $10.6 \mathrm{~g} / \mathrm{dL}$ (range: $6.9-15.5 \mathrm{~g} / \mathrm{L})(p=0.02)$. All the patients received palliative RT alone, as follows: 20 Gy in 5 fractions in $7(28 \%)$ patients, 30 Gy in 10 fractions in 17 (68\%) patients, and $40 \mathrm{~Gy}$ in 20 fractions in 1 (4\%).
The fractionation schema was determined by the condition of the patients and/or the irradiated bladder volume. The median follow-up period after completion of RT was 90 days (range: 11-886 days).

Complete resolution of $\mathrm{MH}$ was achieved in $22 \mathrm{pa}$ tients $(88 \%)$. The median interval from the start of RT to complete resolution of $\mathrm{MH}$ was 9 days (range: 2-179 days). At two weeks after the start of RT, 15 (60\%) patients showed no evidence of MH. Among the three patients who failed to show resolution of the $\mathrm{MH}$, one died of multiple organ failure at the age of 88 years, after surviving for 13 days; the remaining two patients survived for 64 and 105 days, respectively. No significant intergroup differences in the rate of resolution of $\mathrm{MH}$ were found, including between those with and without pretreatment blood transfusion history (7/9 vs. $15 / 16, p=0.53)$. Nine of the $22(41 \%)$ patients who showed resolution of the $\mathrm{MH}$ developed recurrent $\mathrm{MH}$, and the median period to the recurrence of $\mathrm{MH}$ was 129 days (range: 30-692 days). Among the nine patients with recurrent $\mathrm{MH}$, two patients died within a week of recurrence due to severe anemia. The median survival duration of the nine patients was 278 days (range: 60 778 days).

The HFS rate is shown in Figure 1, and the threemonth HFS rate was $52.1 \%$. The duration of HFS per patient is shown in Figure 2. There were significant differences in both the three-month HFS rate between patients with and without a history of pretreatment blood transfusion (HFS rate: $34.6 \%$ vs. $61.5 \%, p=0.03$ ) (Fig. 3). The results of the univariate and multivariate Cox regression

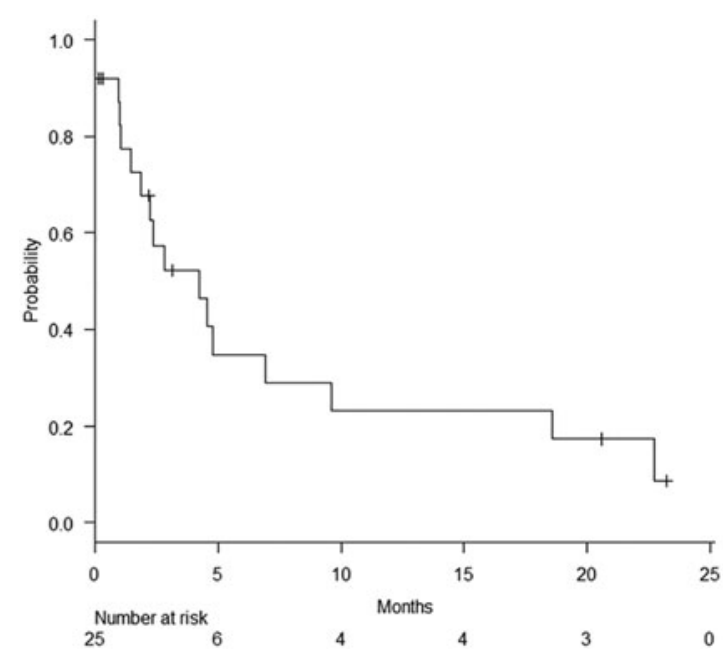

FIG. 1. Hematuria-free survival. 


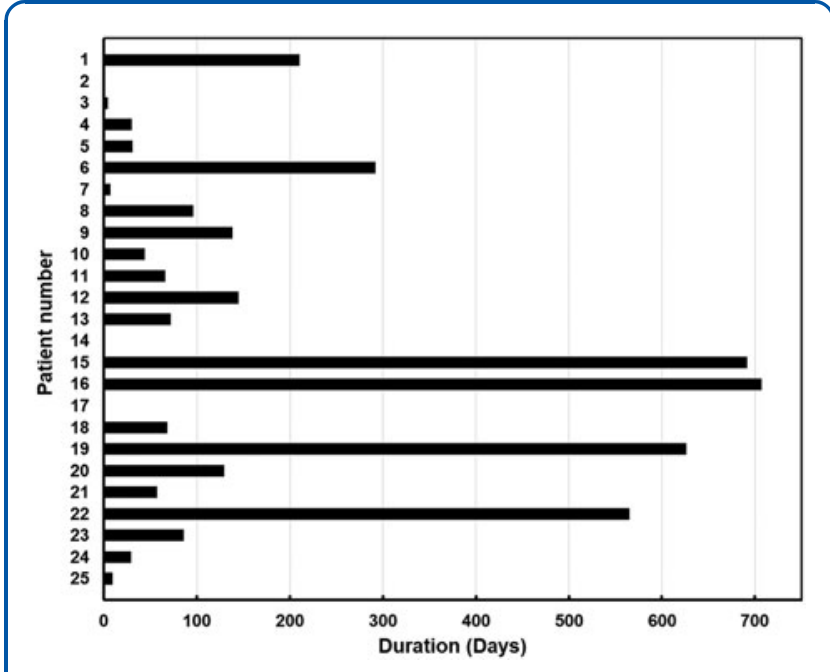

FIG. 2. The duration of hematuria-free survival per patient.

analyses to identify factors influencing the HFS are shown in Table 2. Among these factors, patients with a history of blood transfusion before palliative RT had significantly worse HFS rates $(p=0.01)$. The period from the start of treatment to the day of resolution of $\mathrm{MH}$ was significantly longer in the patients with $\mathrm{T} 3$

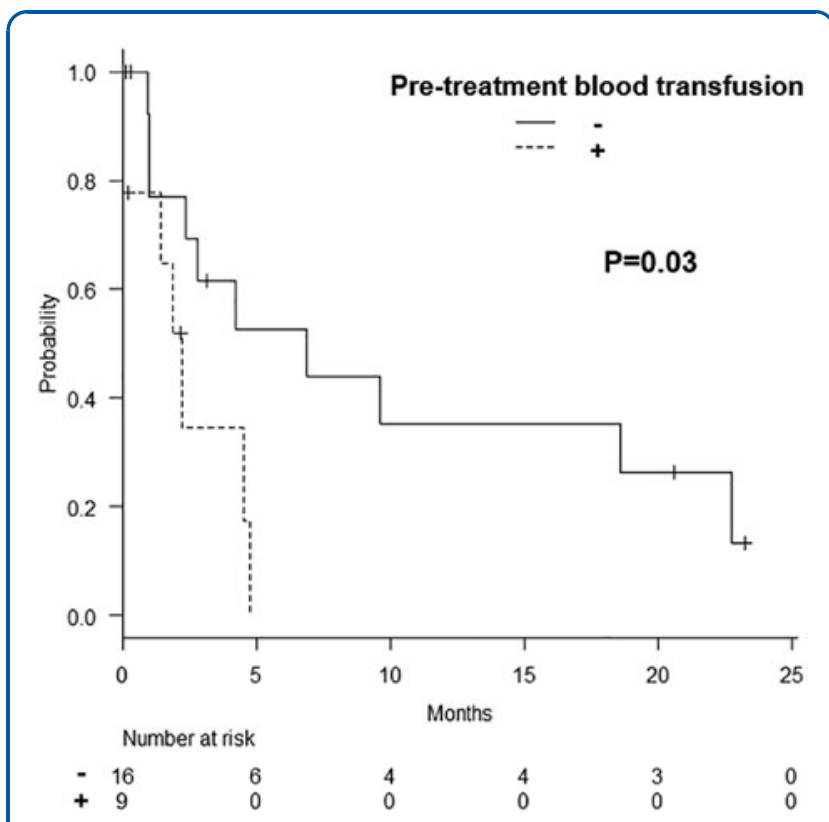

FIG. 3. Hematuria-free survival by the history of pretreatment blood transfusion. and T4 disease than in those with $\mathrm{T} 2$ disease (median duration: 20 and 6 days, respectively; $p=0.01$ ).

None of the patients developed grade 4 or grade 5 toxicities. The toxicities in the 25 patients included 1 (4\%) patient with grade 1 urinary pain, 1 (4\%) patient with grade 1 dermatitis, 3 (12\%) patients with grade 1 diarrhea, and $4(16 \%)$ patients with grade 1 increased urinary frequency. One patient each (4\%) developed grade 2 urinary pain and grade 3 diarrhea.

\section{Discussion}

The results of this retrospective single-institution study indicated that palliative RT may be an effective treatment method to manage $\mathrm{MH}$ caused by tumor recurrence or tumor progression, with acceptable toxicity.

Surgery is the standard treatment of choice for the management of advanced urothelial cancer, ${ }^{10-14}$ while definitive RT with or without chemotherapy has also been used as an alternative approach. ${ }^{15-19}$ However, if as per the tumor board discussion that the cure cannot be expected or the patients refuse the definitive treatment, for these patients, palliative therapy, RT, and palliative surgery are often performed for the purpose of symptom relief and supportive care. ${ }^{5,20}$

Palliative RT has good tolerance and good costperformance characteristics. ${ }^{21,22}$ The main aim of palliative RT is to provide adequate symptomatic relief throughout a patient's anticipated life span. The efficacy of palliative RT for $\mathrm{MH}$ in patients with urological cancers has been reported in several retrospective studies. ${ }^{3,4,6}$ In regard to the mechanism of hemostasis induced by RT, platelet aggregation, injury of vascular endothelial cells, and induction of vascular embolization has been considered the main underlying mechanisms, in addition to tumor shrinkage. ${ }^{23,24}$ To obtain symptomatic relief or hemostasis, a high RT dose, as in definitive RT, is unnecessary. Only one randomizedcontrolled trial, MRC BA09, ${ }^{5}$ has been conducted until date to evaluate the efficacy and toxicity of palliative RT for symptomatic improvement in patients with bladder cancer who are deemed as unsuitable for curative treatment because of comorbidity. In this study, 500 patients from 20 centers received palliative RT at a dose of $35 \mathrm{~Gy}$ in 10 fractions or $21 \mathrm{~Gy}$ in 3 fractions. Symptomatic improvement was noted in $\sim 68 \%$ of patients. Among the patients suffering from hematuria, including microscopic hematuria, $88 \%$ were alleviated. The median time to deterioration of one or more bladder-related symptoms from the start of RT was nine months. 
Table 2. Factors Related to Hematuria-Free Survival Using Cox Regression Analysis

\begin{tabular}{|c|c|c|c|c|c|c|c|}
\hline \multirow[b]{2}{*}{ Factors } & \multicolumn{7}{|c|}{ HFS } \\
\hline & Univariate $(p)$ & Hazard ratio ${ }^{a}$ & $95 \% \mathrm{Cl}^{\mathrm{a}}$ & Multivariate $(p)$ & Hazard ratio ${ }^{b}$ & $95 \%$ & $6 \mathrm{Cl}^{\mathrm{b}}$ \\
\hline Age $(<73 / \geq 73)$ & 0.45 & 0.68 & $0.25-1.85$ & & & & \\
\hline Gender (male/female) & 0.14 & 2.14 & $0.78-5.88$ & - & - & - & - \\
\hline ECOG PS $(0,1 / \geq 2)$ & 0.13 & 0.31 & $0.07-1.40$ & - & - & - & - \\
\hline $\mathrm{Hb}(<9.3 / \geq 9.3 \mathrm{~g} / \mathrm{dL})$ & 0.25 & 0.54 & $0.19-1.54$ & & & & \\
\hline Creatinine $(<0.95 / \geq 0.95 \mathrm{mg} / \mathrm{dL})$ & 0.76 & 0.86 & $0.32-2.30$ & & & & \\
\hline UICC T classifications $(T 1,2 / 3,4)$ & 0.18 & 2.00 & $0.73-5.47$ & - & - & - & - \\
\hline History of pretreatment blood transfusion & 0.04 & 3.16 & $1.04-9.62$ & 0.04 & 3.16 & 1.04 & 9.62 \\
\hline History of chemotherapy before irradiation & 0.09 & 2.67 & $0.87-8.21$ & - & - & - & - \\
\hline Radiation schedule ( $30 \mathrm{~Gy}$ in $10 \mathrm{fr}$. and more/20 Gy/in 5 fr.) & 0.65 & 1.30 & $0.42-1.04$ & & & & \\
\hline
\end{tabular}

${ }^{\mathrm{a}}$ Results from univariate analysis.

${ }^{b}$ Results from multivariate analysis.

—, Variable not included in final step of multivariate analysis; $\mathrm{Cl}$, confidence interval; HFS, hematuria-free survival.

However, the optimal total radiation dose or fractionation schema has not been established, especially for palliative RT to treat $\mathrm{MH}$, due to the limited clinical data available from retrospective studies. In a survey conducted in the Netherlands, nine distinct palliative RT schedules for bleeding tumors were identified, including $1 \times 8$ Gy, $2 \times 8$ Gy, $5 \times 4$ Gy, $5 \times 5$ Gy, and $10-13 \times 3$ Gy. ${ }^{25}$ In Japan, the fractionation schedules for palliative RT used to achieve resolution of $\mathrm{MH}$ varied widely according to the primary tumor sites, such as gastrointestinal and genitourinary tumors, and/or the patients' general condition. The most frequently used fractioned schema was 30 Gy administered in 10 fractions. $^{8}$

In patients in a poor general condition with a limited survival prognosis, hypofractionated RT may be beneficial. $^{4,26}$ Several studies have suggested that shortcourse RT was as efficient as RT administered in a higher number of fractions or long-course RT for obtaining bleeding control. ${ }^{3,5,6,27}$ In a previously conducted retrospective study, 20 Gy in 5 fractions $(n=46), 30 \mathrm{~Gy}$ in 10 fractions $(n=25)$, and $8 \mathrm{~Gy}$ in a single fraction $(n=21)$ were the most commonly used regimens in 112 patients who were receiving palliative $\mathrm{RT}$ for the control of $\mathrm{MH}$ occurring from tumors. ${ }^{27}$ Both longer RT regimens ( $>5$ fractions) and shorter regimens ( $\leq 5$ fractions) exerted equal hemostatic effect $(p=0.497)$ for an equal duration $(p=0.652)$. However, longer regimens caused frequent treatment interruptions and increased hospital days $(22.2 \%$ vs. $5.3 \%$, $p=0.020$ ). Similarly, in the MRC BA09 study, ${ }^{5}$ there was no difference in the survival, symptomatic improvement rate, or toxicity between the two hypofractionated RT schedules ( 35 Gy in 10 fractions vs. $21 \mathrm{~Gy}$ in 3 fractions). Lacarriere et al. ${ }^{6}$ compared two RT schedules retrospectively: the standard treatment arm consisted of $30 \mathrm{~Gy}$ administered in 10 fractions over a period of two weeks, and the study treatment arm consisted of a hypofractionated regimen of 20 Gy administered in 5 fractions over a period of one week to patients with ECOG PS $>2$. No statistically significant difference was observed with respect to the $\mathrm{MH}$ control rate at two weeks after the start of treatment $(54 \%$ vs. $79 \%, p=0.139$ ) or rate of relapse of $\mathrm{MH}$ at six months $(62 \%$ vs. $71 \%){ }^{4}$ Recently, a retrospective study ${ }^{3}$ was conducted in 241 patients from 2 centers to investigate the efficacy of palliative RT in patients with bladder cancer, in which 5 different RT protocols were used: 8 Gy in 1 fraction (11\%), 21 Gy in 3 fractions (15\%), 20 Gy in 5 fractions (18\%), 36 Gy in 6 fractions (36\%), and 27.5 to $30 \mathrm{~Gy}$ in 8 to 10 fractions (18\%). The results showed no significant difference in the clinical outcomes among the five different RT protocols.

In the current study, the resolution of $\mathrm{MH}$ was noted in $88 \%$ of all patients, and $60 \%$ of the patients showed no sign of $\mathrm{MH}$ after two weeks of palliative RT. The duration of hemostasis lasted for about four months. In addition, the toxicity of palliative RT was also acceptable; only one patient developed grade 3 diarrhea. Also, the results of the current study showed that the time to resolution of $\mathrm{MH}$ was longer in the patients with T3 or T4 disease compared with those with T2 disease. The majority of patients who developed recurrent hematuria were patients with $\mathrm{T} 3$ or $\mathrm{T} 4$ disease (7/ $9,78 \%)$. This may indicate the possibility of the need for a higher dose of radiation in patients with more advanced or larger tumors.

Fourteen patients received chemotherapy before palliative RT and all the patients had a 25-day or longer interval before the start of RT. Thus, we considered 
that chemotherapy before irradiation was unlikely to be involved in hemostasis. Otherwise, two patients underwent chemotherapy after the palliative RT. Chemotherapy may have affected the lasting effect of hemostasis in the two treated patients.

Several limitations of this study must be pointed out, including the small sample size and short follow-up time. In addition, the information was obtained retrospectively from the medical record, and date when hemostasis stated is defined as the date of hemostasis. Therefore, it is possible that hemostasis was recorded long after the palliative RT. Therefore, further accumulation of patients is needed to arrive at a more concrete conclusion.

\section{Conclusions}

Our study findings suggest that palliative RT could be an effective and safe treatment option to control macroscopic bleeding in patients with urothelial cancer. RT plays a pivotal role in the management of urothelial cancer, while palliative RT needs more attention. To date, only a handful of studies have shown the effects of palliative RT. Further research is required to determine the optimal individual treatment schedule, and select appropriate candidates for treatment, to guide clinical practice.

\section{Funding Information}

This study was supported, in part, by the Japan Agency for Medical Research and Development (AMED) under grant numbers 18ck0106210h0003 and 19ck0106485h001, and the National Cancer Center Research and Development Fund [grant numbers 31-A-17].

\section{Author Disclosure Statement}

No competing financial interests exist.

\section{References}

1. Siegel RL, Miller KD, Jemal A: Cancer statistics, 2018. CA Cancer J Clin 2018;68:7-30.

2. Abt D, Bywater M, Engeler DS, et al.: Therapeutic options for intractable hematuria in advanced bladder cancer. Int J Urol 2013;20:651-660.

3. Ali $A$, Song YP, Mehta $S$, et al.: Palliative radiation therapy in bladder cancer-importance of patient selection: A retrospective multicenter study. Int J Radiat Oncol Biol Phys 2019;105:389-393.

4. Dirix $P$, Vingerhoedt $S$, Joniau $S$, et al.: Hypofractionated palliative radiotherapy for bladder cancer. Support Care Cancer 2016;24:181-186.

5. Duchesne GM, Bolger JJ, Griffiths GO, et al.: A randomized trial of hypofractionated schedules of palliative radiotherapy in the management of bladder carcinoma: Results of medical research council trial BA09. Int J Radiat Oncol Biol Phys 2000;47:379-388.

6. Lacarriere E, Smaali C, Benyoucef $A$, et al.: The efficacy of hemostatic radiotherapy for bladder cancer-related hematuria in patients unfit for surgery. Int Braz J Urol 2013;39:808-816.
7. McLaren DB, Morrey D, Mason MD: Hypofractionated radiotherapy for muscle invasive bladder cancer in the elderly. Radiother Oncol 1997;43: 171-174.

8. Kosugi T, Shikama N, Saito T, et al.: A nationwide survey in Japan of palliative radiotherapy for bleeding in gastrointestinal and genitourinary tumor patients. World J Oncol 2016;7:29-33.

9. Kanda $Y$ : Investigation of the freely available easy-to-use software 'EZR' for medical statistics. Bone Marrow Transplant 2013;48:452-458.

10. Shariat SF, Palapattu GS, Karakiewicz PI, et al.: Discrepancy between clinical and pathologic stage: Impact on prognosis after radical cystectomy. Eur Urol 2007;51:137-149; discussion 149-151.

11. Wright JL, Lin DW, Porter MP: The association between extent of lymphadenectomy and survival among patients with lymph node metastases undergoing radical cystectomy. Cancer 2008;112:24012408.

12. Margulis V, Shariat SF, Matin SF, et al.: Outcomes of radical nephroureterectomy: A series from the Upper Tract Urothelial Carcinoma Collaboration. Cancer 2009;115:1224-1233.

13. Li WM, Shen JT, Li CC, et al.: Oncologic outcomes following three different approaches to the distal ureter and bladder cuff in nephroureterectomy for primary upper urinary tract urothelial carcinoma. Eur Urol 2010;57: 963-969.

14. Ariane MM, Colin P, Ouzzane A, et al.: Assessment of oncologic control obtained after open versus laparoscopic nephroureterectomy for upper urinary tract urothelial carcinomas (UUT-UCs): Results from a large French multicenter collaborative study. Ann Surg Oncol 2012;19: 301-308.

15. Kaufman DS, Winter KA, Shipley WU, et al.: The initial results in muscle-invading bladder cancer of RTOG 95-06: Phase I/II trial of transurethral surgery plus radiation therapy with concurrent cisplatin and 5-fluorouracil followed by selective bladder preservation or cystectomy depending on the initial response. Oncologist 2000;5:471476.

16. Hagan MP, Winter KA, Kaufman DS, et al.: RTOG 97-06: Initial report of a phase I-II trial of selective bladder conservation using TURBT, twice-daily accelerated irradiation sensitized with cisplatin, and adjuvant MCV combination chemotherapy. Int J Radiat Oncol Biol Phys 2003;57:665672.

17. Efstathiou JA, Bae K, Shipley WU, et al.: Late pelvic toxicity after bladdersparing therapy in patients with invasive bladder cancer: RTOG 89-03, 9506, 97-06, 99-06. J Clin Oncol 2009;27:4055-4061.

18. Kaufman DS, Winter KA, Shipley WU, et al.: Phase I-II RTOG study (99-06) of patients with muscle-invasive bladder cancer undergoing transurethral surgery, paclitaxel, cisplatin, and twice-daily radiotherapy followed by selective bladder preservation or radical cystectomy and adjuvant chemotherapy. Urology 2009;73:833-837.

19. Mitin T, Hunt D, Shipley WU, et al.: Transurethral surgery and twice-daily radiation plus paclitaxel-cisplatin or fluorouracil-cisplatin with selective bladder preservation and adjuvant chemotherapy for patients with muscle invasive bladder cancer (RTOG 0233): A randomised multicentre phase 2 trial. Lancet Oncol 2013;14:863-872.

20. Flaig TW, Spiess PE, Agarwal N, et al.: Bladder Cancer, Version 3.2020 , NCCN Clinical Practice Guidelines in Oncology. J Natl Compr Canc Netw 2020;18:329-354.

21. Lutz ST, Jones J, Chow E: Role of radiation therapy in palliative care of the patient with cancer. J Clin Oncol 2014;32:2913-2919.

22. Park KR, Lee CG, Tseng YD, et al.: Palliative radiation therapy in the last 30 days of life: A systematic review. Radiother Oncol 2017;125:193199.

23. Krigsfeld GS, Savage AR, Billings PC, et al.: Evidence for radiationinduced disseminated intravascular coagulation as a major cause of radiation-induced death in ferrets. Int J Radiat Oncol Biol Phys 2014;88: 940-946.

24. Wang Y, Boerma M, Zhou D: lonizing radiation-induced endothelial cell senescence and cardiovascular diseases. Radiat Res 2016;186: 153-161.

25. Strijbos J, van der Linden $\mathrm{YM}$, Vos-Westerman $\mathrm{H}$, et al.: Patterns of practice in palliative radiotherapy for bleeding tumours in the Netherlands; a survey study among radiation oncologists. Clin Transl Radiat Oncol 2019; 15:70-75.

26. Kouloulias V, Tolia M, Kolliarakis N, et al.: Evaluation of acute toxicity and symptoms palliation in a hypofractionated weekly schedule of external 
radiotherapy for elderly patients with muscular invasive bladder cancer. Int Braz J Urol 2013;39:77-82.

27. Sapienza LG, Ning MS, Jhingran A, et al.: Short-course palliative radiation therapy leads to excellent bleeding control: A single centre retrospective study. Clin Transl Radiat Oncol 2019;14:40-46.

Cite this article as: Zhang $\mathrm{H}$, Hojo $\mathrm{H}$, Vijay Parshuram R, Nakamura $\mathrm{N}$, Nakamura M, Okumura M, Hirano Y, Motegi A, Kageyama S-I, Zenda S, Akimoto T (2020) Palliative radiation therapy for macroscopic hematuria caused by urothelial cancer, Palliative Medicine Reports 1:1, 201207, DOI: 10.1089/pmr.2020.0027.

\section{Abbreviations Used}

CTV = clinical target volume

ECOG = Eastern Cooperative Oncology Group

$\mathrm{HFS}=$ hematuria-free survival

$\mathrm{MH}=$ macroscopic hematuria

PS = performance status

PTV $=$ planning target volume

$\mathrm{RT}=$ radiation therapy

$\mathrm{UC}=$ urothelial carcinoma

UICC $=$ Union for International Cancer Control
Publish in Palliative Medicine Reports

Palliative

Medicine

Reports

- Immediate, unrestricted online access

- Rigorous peer review

- Compliance with open access mandates

- Authors retain copyright

- Highly indexed

- Targeted email marketing

\section{liebertpub.com/pmr}

